\title{
ON MODULES OF FINITE PROJECTIVE DIMENSION
}

\author{
S. P. DUTTA
}

\begin{abstract}
We address two aspects of finitely generated modules of finite projective dimension over local rings and their connection in between: embeddability and grade of order ideals of minimal generators of syzygies. We provide a solution of the embeddability problem and prove important reductions and special cases of the order ideal conjecture. In particular, we derive that, in any local ring $R$ of mixed characteristic $p>0$, where $p$ is a nonzero divisor, if $I$ is an ideal of finite projective dimension over $R$ and $p \in I$ or $p$ is a nonzero divisor on $R / I$, then every minimal generator of $I$ is a nonzero divisor. Hence, if $P$ is a prime ideal of finite projective dimension in a local ring $R$, then every minimal generator of $P$ is a nonzero divisor in $R$.
\end{abstract}

In this note we consider two aspects of finitely generated modules of finite projective dimension over any local ring: embeddability and grade of order ideals of minimal generators of its syzygies (minimal). In regard to embeddability, Auslander and Buchweitz [1] proved that any finitely generated module on a Gorenstein local ring can be embedded in a module of finite projective dimension such that the cokernel is Cohen-Macaulay. This result has several applications in solving homological questions and conjectures in commutative algebra, including Serre's $\chi_{i}$-conjectures and their generalizations on intersection multiplicity (see [5]). For all these conjectures, one is usually concerned with finitely generated modules $M$ which are of finite projective dimension over a local ring $R$ but are not so over $R / x R, x$ being a nonzero divisor in the annihilator of $M$ in $R$. However, a similar result has been absent for non-Gorenstein local rings until now. In this note we prove the following with respect to embeddability for modules of finite projective dimension.

TheOREM 1. Let $(R, m)$ be a local ring, and let $M$ be a finitely generated module of finite projective dimension with positive grade over $R$.

Received March 4, 2013. Revised March 5, 2014. Accepted August 13, 2014.

2010 Mathematics Subject Classification. Primary 13D02, 13D22; Secondary 13C15, 13D25, $13 \mathrm{H} 05$. 
Let $\mathbf{x}=\left\{x_{1}, \ldots, x_{t}\right\}$ be any $R$-sequence contained in the annihilator of $M$ (henceforth $\operatorname{ann}_{R} M$ ), and let $\bar{R}=R /\left(x_{1}, \ldots, x_{t}\right)$. Then there exists a short exact sequence of finitely generated $\bar{R}$-modules

$$
0 \rightarrow M \rightarrow Q \rightarrow T \rightarrow 0
$$

where $\operatorname{pd}_{\bar{R}} Q<\infty$ and $\operatorname{pd}_{R} T=t$.

From the construction of $Q$, it would follow that support of $Q$ over $\bar{R}=\operatorname{Spec}(\bar{R})$ and $Q$ satisfies the strong intersection conjecture due to Peskine and Szpiro [15]. Since $T$ is perfect, $T$ also possesses the above property. The effect of this theorem on generalizations of Serre's conjectures on intersection multiplicities for arbitrary local rings, in particular for CohenMacaulay rings, will be the subject of a future paper. This paper focuses on its effect on the order ideal conjecture, which is our next topic.

The order ideal conjecture stems from Evans and Griffith's work [7] on grade of order ideals of minimal generators of syzygies in equicharacteristic. The statement of the conjecture is the following.

Order Ideal Conjecture. Let $(R, m)$ be a local ring. Let $M$ be a finitely generated module of finite projective dimension over $R$, and let $S_{i}$ denote its ith syzygy for $i>0$. If $\beta$ is a minimal generator of $S_{i}$, then the order ideal $\mathcal{O}_{S_{i}}(\beta)$ has grade at least $i$.

Let us recall that $\mathcal{O}_{S_{i}}(\beta)=\left\{f(\beta) \mid f \in \operatorname{Hom}_{R}\left(S_{i}, R\right)\right\}$.

We say that a module $M$ satisfies the order ideal conjecture if order ideals of minimal generators of all its syzygies satisfy the respective grade inequalities mentioned above.

Evans and Griffith [7], [9] proved the above conjecture for equicharacteristic local rings in order to solve the syzygy problem over the above class of rings. The existence of big Cohen-Macaulay modules, due to Hochster [12], played an important role in their proof. Later in [10] they proved a graded version of the above conjecture for a certain class of graded rings in mixed characteristic. We also refer the reader to [3, Theorem 9.5.2] for a more general version of the order ideal theorem in the equicharacteristic case. Actually, for their proof of the syzygy theorem in equicharacteristic, Evans and Griffith needed to prove the above conjecture only for modules which are locally free on the punctured spectrum of $\operatorname{spec}(R), R$ being regular local. And they reduced the proof of this case to what is now known as the improved new intersection conjecture. Later Hochster [13] showed 
that the canonical element conjecture implies the improved new intersection conjecture. The equivalence of these two conjectures was established in [4]. In our most recent work [6], we have shown that a particular case of the order ideal conjecture implies the monomial conjecture and therefore also implies all its equivalent forms - for example, the direct summand conjecture, the canonical element conjecture, and the improved new intersection conjecture. Thus, the order ideal conjecture now occupies a central position among several homological conjectures in commutative algebra.

First, let us mention that in order to prove the order ideal conjecture on arbitrary local rings $R$, it is enough to concentrate on first syzygies of modules of finite projective dimension (see Lemma 2.1). Theorem 2.3 shows that for the validity of the order ideal conjecture it is enough to prove that every minimal generator of ideals of height 2, grade 2, and of finite projective dimension over $R$ is a nonzero divisor. In Theorem 2.5, we prove the following: Given a module of finite projective dimension $M$ on a local ring $R$, there exists an $R$-sequence $x_{1}, \ldots, x_{h}, h=\operatorname{pd}_{R} M$, such that for any $R$-module $N$ for which $x_{1}, \ldots, x_{h}$ form an $N$-sequence, $\operatorname{Tor}_{j}^{R}(M, N)=0$ for $j>0$.

This theorem leads us to the following (see Corollary 2.7).

Let $(R, m)$ be a local ring, and let $M$ be a finitely generated module of finite projective dimension over $R$. Let $x_{1}, \ldots, x_{h}$ be an $R$-sequence as mentioned above. If $R$ has mixed characteristic $p>0$, we assume that $p, x_{1}, \ldots, x_{h}$ form a part of a system of parameters of $R$. Then for every minimal generator $\beta$ of $S_{1}=\operatorname{Syz}_{R}^{1}(M)$, the grade of $\mathcal{O}_{S_{1}}(\beta) \geq 1$.

The statement of our main theorem in Section 2 is the following.

THEOREM 2.11. Let $(R, m)$ be a local ring of mixed characteristic $p>0$. Let $M$ be a finitely generated module of finite projective dimension over $R$, and let $\beta$ be a minimal generator of $S_{i}$, the ith syzygy (minimal) of $M$, for $i>0$. We assume that either $p$ is a nonzero divisor in $R$ or $p$ is nilpotent. We have the following.

(a) If $p$ is nilpotent, then the order ideal conjecture is valid on $R$.

(b) If $p M=0$, then the grade of $\mathcal{O}_{S_{1}}(\beta) \geq 1$.

(c) Suppose that balanced or complete almost Cohen-Macaulay algebras exist over complete local domains. If $p M=0$ and $p \in m-m^{2}$, then the grade of $\mathcal{O}_{S_{i}}(\beta) \geq i, \forall i \geq 1$. 
(d) Assume that every element in $m-m^{2}$ is a nonzero divisor and that the order ideal conjecture is valid over $R / x R$ for $x \in m-m^{2}$. If $\operatorname{ann}_{R} M \cap$ $\left(m-m^{2}\right) \neq \phi$ or $\operatorname{depth}_{R} M>0$, then $\operatorname{grade} \mathcal{O}_{S_{i}}(\beta) \geq i, \forall i \geq 1$.

As a consequence of the above theorem, we have the following corollaries.

Corollary 2.12. For any ideal I of finite projective dimension over $R$ of mixed characteristic $p>0$, where $p$ is a nonzero divisor in $R$, if $p \in I$ or $p$ is a nonzero divisor on $R / I$, then every minimal generator of $I$ is a nonzero divisor. In particular, if $P$ is a prime ideal of finite projective dimension over $R$, then every minimal generator of $P$ is a nonzero divisor in $R$.

COROllary 2.13. Let $(R, m)$ be a regular local ring of dimension $n$, and assume that the order ideal conjecture is valid for regular local rings of dimension $(n-1)$. If $M$ is a finitely generated $R$-module such that either $M$ is annihilated by a regular parameter or $\operatorname{depth}_{R} M>0$, then $M$ satisfies the order ideal conjecture.

The two main ingredients of our proof of Theorem 2.11 are Theorem 1.2 and Shimomoto's theorem (Theorem 2.10) on the existence of almost CohenMacaulay algebras (see [17, Theorem 5.3]).

Throughout this note, local means Noetherian local, $\operatorname{pd}_{R} M$ denotes projective dimension of $M$ over $R$, and $\operatorname{Syz}_{R}^{i}(M)$ denotes the $i$ th syzygy in a minimal free resolution of $M$. (For definitions of standard notions and their basic properties, see [3].)

\section{$\S 1$.}

First we would like to mention the following proposition.

Proposition 1.1 ([4, Proposition 1.1]). Let $A$ be a local ring, and let $F_{\bullet}: \rightarrow A^{s_{i}} \rightarrow A^{s_{i-1}} \rightarrow \cdots \rightarrow A^{s_{0}} \rightarrow 0$ be a free complex with $H_{0}\left(F_{\bullet}\right)=M$. Let $N \subset M$ be a submodule of $M$. Then we can construct a free complex $G_{\bullet}: \rightarrow A^{d_{i}} \rightarrow A^{d_{i-1}} \rightarrow \cdots \rightarrow A^{d_{0}} \rightarrow 0$ with $H_{0}(G)=N$ and a map $\phi_{\bullet}: G_{\bullet} \rightarrow$ $F_{\bullet}$ such that $\phi_{0}$ induces the inclusion $N \hookrightarrow M$ and such that the mapping cone of $\phi_{\bullet}$ is a free resolution of $M / N$; that is, $\phi_{\bullet}$ induces an isomorphism $H_{i}\left(G_{\bullet}\right) \stackrel{\sim}{\longrightarrow} H_{i}\left(F_{\bullet}\right)$ for $i>0$. Moreover, by our construction, $G_{\bullet}$ is minimal.

For a proof we refer the reader to [4, Proposition 1.1].

Next we prove the main theorem of this section. 
THEOREM 1.2. Let $(R, m)$ be a local ring, and let $M$ be a finitely generated module of finite projective dimension over $R$. Assume that grade $_{R} M>0$. Let $\mathbf{x}$ denote the ideal generated by an $R$-sequence of length $t$ contained in $\operatorname{ann}_{R} M$, and let $\bar{R}=R / \mathbf{x} R$. Then there exists a short exact sequence of finitely generated $\bar{R}$-modules

$$
0 \rightarrow M \rightarrow Q \rightarrow T \rightarrow 0
$$

such that $\operatorname{pd}_{\bar{R}} Q<\infty$ and $\operatorname{pd}_{R} T=t$.

Proof. First suppose that $\operatorname{pd}_{R} M=1$. Let us recall that $\operatorname{grade}_{R} M \leq$ $\operatorname{pd}_{R} M$. Since $\operatorname{grade}_{R} M>0$, it follows that $\operatorname{grade}_{R} M=1$. Let $x \in \operatorname{ann}_{R} M$ be a nonzero divisor. Consider a minimal free resolution: $0 \rightarrow R^{t_{1}} \stackrel{f}{\longrightarrow} R^{t_{0}} \rightarrow$ $M \rightarrow 0$ of $M$ over $R$. Tensoring this resolution with $R / x R$, we obtain an exact sequence: $0 \rightarrow \operatorname{Tor}_{1}^{R}(M, R / x R) \rightarrow(R / x R)^{t_{1}} \stackrel{\bar{f}}{\longrightarrow}(R / x R)^{t_{0}} \rightarrow M \rightarrow 0$. Since $x \in \operatorname{ann}_{R} M$, $\operatorname{Tor}_{1}^{R}(M, R / x R) \simeq M$. Let $T=\operatorname{im} \bar{f}$. Hence, we obtain the short exact sequence $0 \rightarrow M \rightarrow(R / x R)^{t_{1}} \rightarrow T \rightarrow 0$, and our assertion follows. So we can assume that $\operatorname{pd}_{R} M \geq 2$.

Let $\left(F_{\bullet}, d_{\bullet}\right): 0 \rightarrow R^{r_{n}} \stackrel{d_{n}}{\longrightarrow} R^{r_{n-1}} \rightarrow \cdots \rightarrow R^{r_{1}} \stackrel{d_{1}}{\longrightarrow} R^{r_{0}} \rightarrow 0$ be a minimal projective resolution of $M$ over $R$, and let $\left(L_{\bullet}, \phi_{\bullet}\right): \rightarrow \bar{R}^{s_{n}} \stackrel{\phi_{n}}{\longrightarrow} \bar{R}^{s_{n-1}} \rightarrow$ $\cdots \rightarrow \bar{R}^{s_{1}} \stackrel{\phi_{0}}{\longrightarrow} \bar{R}^{s_{0}} \rightarrow 0$ be a minimal projective resolution of $M$ over $\bar{R}$. Since $\operatorname{pd}_{R} M=n$, grade $\operatorname{Ext}_{R}^{i}(M, R) \geq i$ for $1 \leq i \leq n, \operatorname{Ext}_{R}^{n}(M, R) \neq 0$, and $\operatorname{Ext}_{R}^{i}(M, R)=0$ for $i>n$. Hence, $\operatorname{grade}_{\operatorname{Ext}} \frac{i}{R}(M, \bar{R}) \geq i$ for $1 \leq i \leq n-t$, $\operatorname{Ext}_{\bar{R}}^{n-t}(M, \bar{R}) \neq 0\left(\simeq \operatorname{Ext}_{R}^{n}(M, R)\right)$, and $\operatorname{Ext}_{\bar{R}}(M, \bar{R})=0$ for $i>(n-t)$.

Applying $\operatorname{Hom}_{\bar{R}}(-, \bar{R})$ to $L_{\bullet}$, we obtain the following free complex $L_{\bullet}^{*}$ :

$$
L_{\bullet}^{*}: 0 \rightarrow \bar{R}^{s_{0}^{*}} \rightarrow \bar{R}^{s_{1}^{*}} \rightarrow \cdots \rightarrow \bar{R}^{s_{n-t}^{*}} \rightarrow 0 .
$$

(For any $R(\bar{R})$ module $V, V^{*}=\operatorname{Hom}_{R}(M, R)\left(\operatorname{Hom}_{\bar{R}}(V, \bar{R})\right)$.)

Let $G=\operatorname{Coker} \phi_{n-t}^{*}$. We have a short exact sequence

$$
0 \rightarrow \operatorname{Ext}_{\bar{R}}^{n-t}(M, \bar{R}) \stackrel{c}{\longrightarrow} G \stackrel{\eta}{\longrightarrow} \operatorname{Im} \phi_{n-t+1}^{*} \rightarrow 0 .
$$

By the above proposition, there exists a minimal free complex $\left(P_{\bullet}, \alpha_{\bullet}\right): \rightarrow$ $\bar{R}^{b_{n-t}} \stackrel{\alpha_{n-t}}{\longrightarrow} \bar{R}^{b_{n-t-1}} \rightarrow \cdots \stackrel{\alpha_{1}}{\longrightarrow} \bar{R}^{b_{0}} \rightarrow 0$ with $H_{0}\left(P_{\bullet}\right)=\operatorname{Ext}_{\bar{R}}^{n-t}(M, \bar{R})$ and a map $\Psi_{\bullet}: P_{\bullet} \rightarrow L_{\bullet}^{*}$ such that $\Psi_{\bullet}$ induces the injection $c$ in (1) and the mapping cone of $\Psi_{\bullet}$ is a free resolution of $\operatorname{Im} \phi_{n-t+1}^{*}$. We have the following 
commutative diagram:

(2)

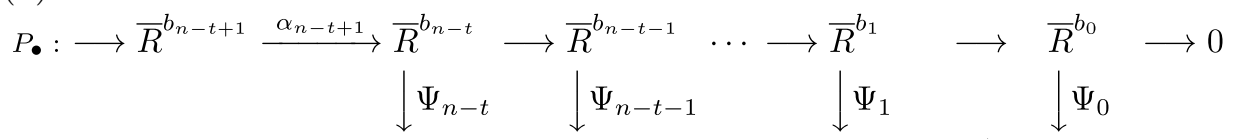

$$
\begin{aligned}
& L_{\bullet}^{*}: \quad 0 \quad \longrightarrow \quad \bar{R}^{s_{0}^{*}} \longrightarrow \bar{R}^{s_{1}^{*}} \quad \cdots \longrightarrow \bar{R}^{s_{n-t-1}^{*}} \stackrel{\phi_{n-t}^{*}}{\longrightarrow} \bar{R}^{s_{n-t}^{*}} \longrightarrow 0
\end{aligned}
$$

Applying $\operatorname{Hom}(-, \bar{R})$ to $(2)$, we obtain the following commutative diagram of exact complexes:

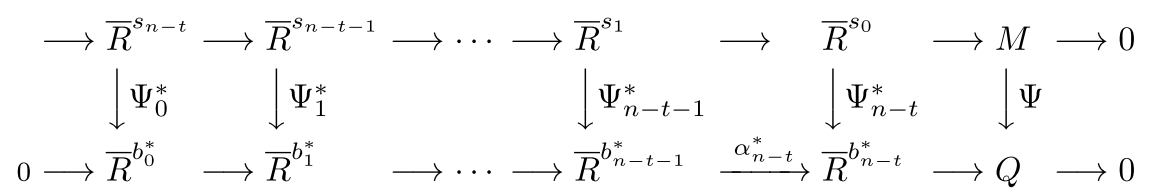

where $Q=$ Coker $\alpha_{n-t}^{*}$ and $\Psi: M \rightarrow Q$ is induced by $\Psi_{n-t}^{*}$. Since grade $\operatorname{Ext}_{\bar{R}}(M, \bar{R}) \geq i, 1 \leq i \leq n-t$, the bottom row of (3) provides a minimal free resolution of $Q$, and hence $\operatorname{pd}_{\bar{R}} Q=n-t$. Moreover, by construction, $\operatorname{Ext}_{\bar{R}}(Q, \bar{R}) \stackrel{\sim}{\longrightarrow} \operatorname{Ext}_{\bar{R}}(M, \bar{R})$ for $i>0$.

Next we want to prove that $\Psi: M \rightarrow Q$ in (3) is injective.

Let $\bar{F}_{\bullet}=F_{\bullet} \otimes \bar{R}, S_{i}=\operatorname{Syz}_{R}^{i}(M)$, and let $\bar{S}_{i}=S_{i} / \mathbf{x} S_{i}$, for $i>0$. Since $\mathbf{x}$ is generated by an $R$-sequence of length $t$ in $\operatorname{ann}_{R} M$, we have $\operatorname{Tor}_{t}^{R}(M, \bar{R}) \stackrel{\sim}{\longleftarrow} M$ and $\operatorname{Tor}_{i}^{R}(M, \bar{R})=0$ for $i>t$. Tensoring the exact sequence $0 \rightarrow S_{t} \rightarrow R^{r_{t-1}} \rightarrow S_{t-1} \rightarrow 0$ by $\bar{R}$, we obtain an exact sequence

$$
0 \rightarrow M \stackrel{j}{\longrightarrow} \bar{S}_{t} \rightarrow \bar{R}^{r-1} \rightarrow \bar{S}_{t-1} \rightarrow 0
$$

Let $\theta_{\bullet}: L_{\bullet} \rightarrow \bar{F} \bullet$ be a lift of $j: M \hookrightarrow \bar{S}_{t}$. We have the following commutative diagram:

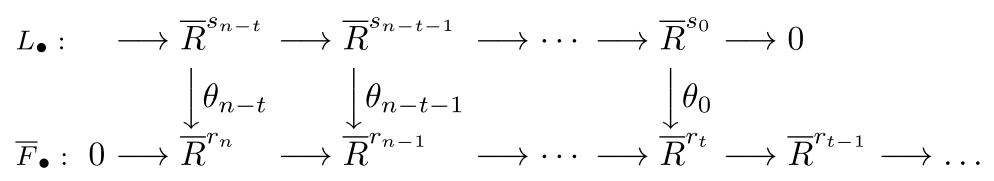

The mapping cone of $\left\{\theta_{\bullet}\right\}$ in (4) is a free resolution of $\operatorname{Im} \bar{d}_{t}$ over $\bar{R}$, and $\theta_{0}$ induces the isomorphism $\tilde{\theta}_{0}: M \stackrel{\sim}{\longrightarrow} \operatorname{Tor}_{t}^{R}(M, \bar{R})$ via $j: M \hookrightarrow \bar{S}_{t}$. 
Applying $\operatorname{Hom}(-, \bar{R})$ to (4), the following commutative diagram is obtained:

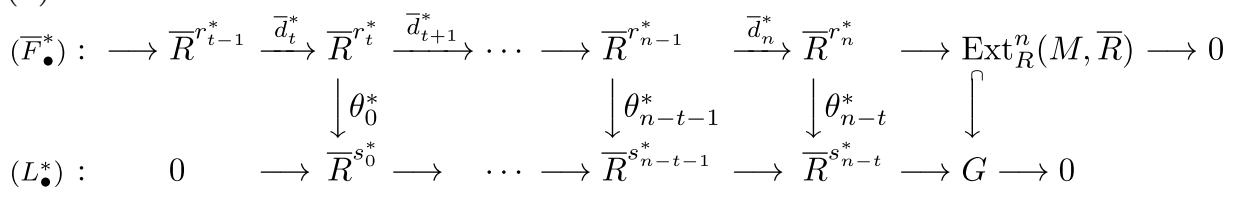

Since the mapping cone of (4) is a free resolution of $\operatorname{Im} \bar{d}_{t}$ over $\bar{R}$ and $\operatorname{Ext}_{\bar{R}}(M, \bar{R})=0$ for $i>n-t, \theta_{n-t}^{*}$ induces an isomorphism $\operatorname{Ext}_{R}^{n}(M, \bar{R}) \stackrel{\sim}{\longrightarrow}$ $\operatorname{Ext}_{\bar{R}}^{n-t}(M, \bar{R})$. Hence, the inclusion $\operatorname{Ext}_{R}^{n}(M, \bar{R}) \hookrightarrow G$ in (5) can be identified with $c: \operatorname{Ext}_{\bar{R}}-t(M, \bar{R}) \hookrightarrow G$ in $(1)$.

Let $\left(H_{\bullet}, \delta_{\bullet}\right)$ denote the mapping cone of $\Psi_{\bullet}$ in (2). By construction this is a free resolution of $\operatorname{Im} \phi_{n-t+1}^{*}$. Let $\eta_{\bullet}: L_{\bullet}^{*} \rightarrow H_{\bullet}\left(\eta_{i}: \bar{R}^{s_{i}^{*}} \rightarrow \bar{R}^{b_{n-i-t-1}} \oplus \bar{R}^{s_{i}^{*}}\right)$, $\gamma_{\bullet}: H_{\bullet} \rightarrow P_{\bullet}(-1)\left(\gamma_{n-j}: \bar{R}^{b_{n-j}} \oplus \bar{R}^{s_{j-t-1}^{*}} \rightarrow \bar{R}^{b_{n-j}}\right)$ denote the corresponding inclusion and projection maps, respectively. Then $\eta_{\bullet} \cdot \theta_{\bullet}^{*}: \bar{F}_{\bullet}^{*} \rightarrow H_{\bullet}$ lifts the composite $\eta \cdot c: \operatorname{Ext}_{\bar{R}}^{n-t}(M, \bar{R}) \stackrel{c}{\longrightarrow} G \stackrel{\eta}{\longrightarrow} \operatorname{Im} \phi_{n-t+1}^{*}$. Since $\eta \cdot c=0$ and $\left(H_{\bullet}, \delta_{\bullet}\right)$ is a free resolution of $\operatorname{Im} \phi_{n-t+1}^{*}, \eta_{\bullet} \cdot \theta_{\bullet}^{*}$ is homotopic to 0. Hence, there exist homotopy maps $h_{\bullet}: \bar{F}_{\bullet}^{*} \rightarrow H_{\bullet}, h_{j}: \bar{R}^{r_{j}^{*}} \rightarrow \bar{R}^{b_{n-j}} \oplus \bar{R}^{s_{j-t-1}^{*}}$, for $t \leq j \leq n$, such that

$$
\delta_{n-j} \cdot h_{j}+h_{j+1} \cdot \bar{d}_{j+1}^{*}=\eta_{j-1} \cdot \theta_{j-1}^{*}
$$

Let $\beta_{\bullet}=\gamma_{\bullet} \cdot h_{\bullet},\left\{\beta_{j}=\gamma_{n-j} \cdot h_{j}\right\}$. Then $\beta_{\bullet}^{\prime}=\left\{(-1)^{j+1} \beta_{j}\right\}: \bar{F}_{\bullet}^{*} \rightarrow P_{\bullet}$ is a map of complexes. Consider $\Psi_{\bullet} \cdot \beta_{\bullet}^{\prime}: F_{\bullet}^{*} \rightarrow L_{\bullet}^{*}$.

For $1 \leq j \leq n$, let $k_{j}=\pi_{2} \cdot h_{j}: \bar{R}^{r_{j}^{*}} \rightarrow \bar{R}^{s_{j-t-1}^{*}}$, where $\pi_{2}: \bar{R}^{b_{n-j}} \oplus$ $\bar{R}^{s_{j-t-1}^{*}} \rightarrow \bar{R}^{s_{j-t-1}^{*}}$ is the projection on the second component. It can be checked that, for $t \leq j \leq n$,

$$
\theta_{j-1}^{*}-(-1)^{n-j-1} \Psi_{n-j} \cdot \beta_{j}=\phi_{j-1}^{*} \cdot k_{j}+k_{j+1} \cdot \bar{d}_{j+1}^{*}
$$

that is, $\theta_{\bullet}^{*}$ and $\Psi_{\bullet} \beta_{\bullet}^{\prime}$ are homotopic.

The following commutative diagrams are provided to clarify (6) and (7) for $t=1$ : 
(8)
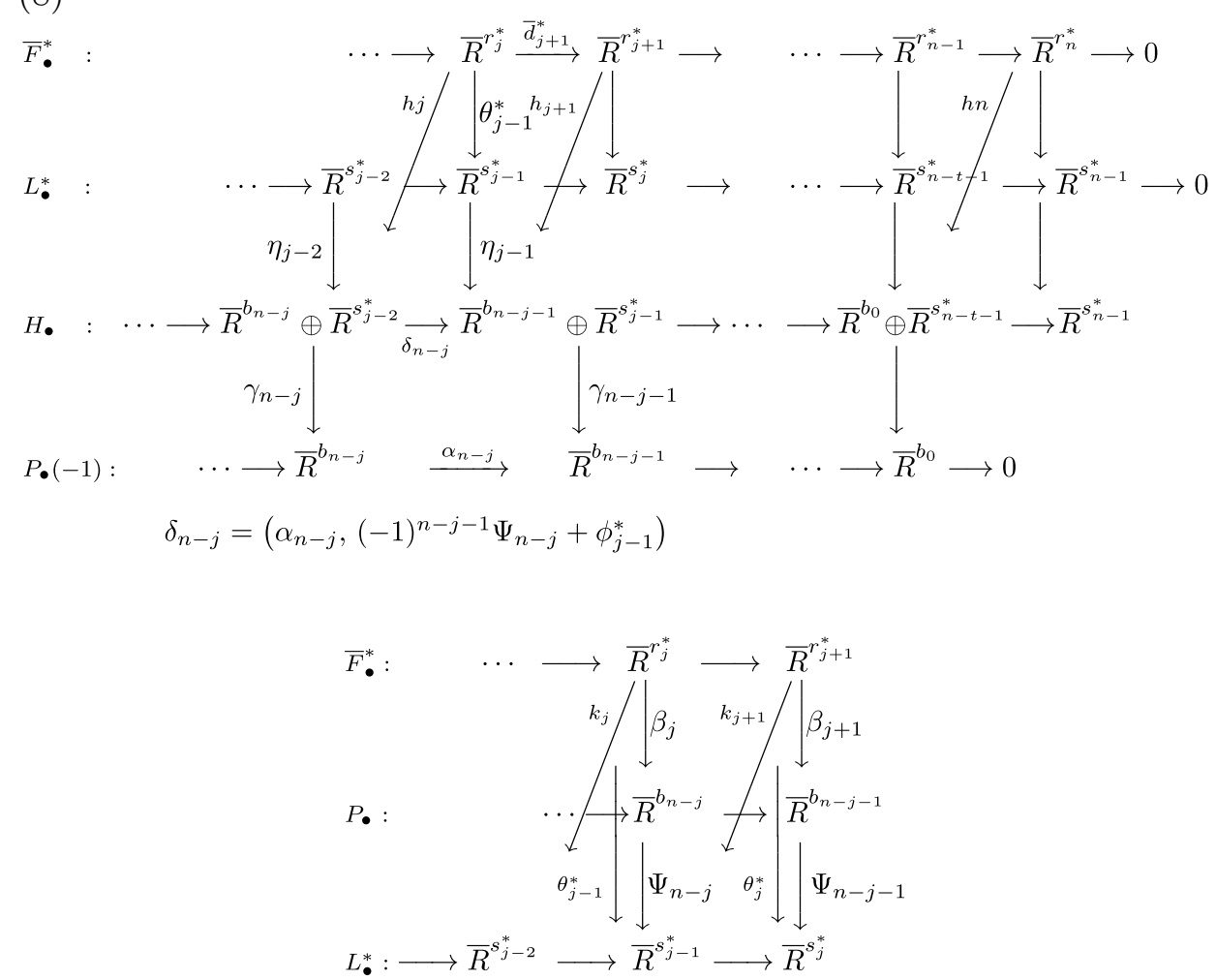

Hence, $\left\{\theta_{\bullet}^{*}\right\}$ and $\left\{\Psi_{\bullet} \beta_{\bullet}^{\prime}\right\}$ define identical maps (modulo \pm sign) on homologies of $F_{\bullet}^{*}$ and $L_{\bullet}$ and, consequently, for the homologies of their respective duals. If $\tilde{\Psi}: M \rightarrow H^{n-t}\left(P_{\bullet}^{*}\right), \tilde{\beta}: H^{n-t}\left(P_{\bullet}^{*}\right) \rightarrow \operatorname{Tor}_{t}^{R}(M, \bar{R})$, and $\tilde{\theta}_{0}: M \stackrel{\sim}{\longrightarrow}$ $\operatorname{Tor}_{t}^{R}(M, \bar{R})$ denote the maps induced by $\Psi_{n-t}^{*}, \beta_{t}^{*}$, and $\left(\theta_{0}^{*}\right)^{*}=\theta_{0}$, respectively, then $\tilde{\theta}_{0}=\tilde{\beta} \cdot \tilde{\Psi}$ (modulo a sign). Since $\tilde{\theta}_{0}$ is an isomorphism, $\Psi=$ (inclusion of $\left.H^{n-t}\left(P_{\bullet}^{*}\right) \hookrightarrow Q\right) \cdot \tilde{\Psi}: M \hookrightarrow Q$ is injective.

Let Coker $\psi=T$; then $0 \rightarrow M \stackrel{\Psi}{\longrightarrow} Q \rightarrow T \rightarrow$ is exact. Since the mapping cones of $\Psi_{\bullet}^{*}, \Psi_{\bullet}$ are free resolutions of $T, \operatorname{Im} \phi_{n-t+1}^{*}$, respectively, and $\operatorname{Ext}_{\bar{R}}(M, \bar{R}) \stackrel{\sim}{\longrightarrow} \operatorname{Ext}_{\bar{R}}(Q, \bar{R})$ for $i>0$, it follows that $\operatorname{Ext}_{\bar{R}}(T, \bar{R})=0$ for $i>0$; that is, $\operatorname{Ext}_{R}^{i}(T, R)=0$ for $i>t$. Since $\operatorname{pd}_{R} M<\infty$, we have $\operatorname{pd}_{R} T<\infty$, and hence $\operatorname{pd}_{R} T=t$. This completes our proof.

Corollary 1.3. Let $R$ be a local ring, and let $M$ be a finitely generated module of finite projective dimension with $\operatorname{pd}_{R} M>1$ and grade $R>0$. Given any nonzero divisor $x \in \operatorname{ann}_{R} M, M$ can be embedded in a finitely generated module $Q$ of finite projective dimension over $R / x R$ in such a 
way that, if $\left(G_{\bullet}, \gamma_{\bullet}\right),\left(F_{\bullet}, d_{\bullet}\right)$ are minimal free resolutions of $M$ and $Q$ over $R$, respectively, and $\phi_{\bullet}: G_{\bullet} \rightarrow F_{\bullet}$ is a lift of $i: M \hookrightarrow Q$, then $\phi_{\bullet}$ induces our isomorphism between $\left(G_{\bullet}, \gamma_{\bullet}\right)_{i \geq 2}$ and $\left(F_{\bullet}, d_{\bullet}\right)_{i \geq 2}, \phi_{1}\left(G_{1}\right)$ is either a summand of or isomorphic to $F_{1}$, and $\phi_{0}$ is an injection. Moreover, $\operatorname{syz}_{R}^{1}(M) \oplus R^{t} \simeq \operatorname{syz}_{R}^{1}(Q)$ for some $t \geq 0$.

Proof. By Theorem 1.2 we have an exact sequence of $R / x R$ modules

$$
0 \rightarrow M \stackrel{i}{\rightarrow} Q \stackrel{\eta}{\longrightarrow} T \rightarrow 0
$$

where $\operatorname{pd}_{R / x R} Q<\infty$ and $\operatorname{pd}_{R} T=1$.

The proof of the corollary now follows directly either by constructing a minimal free resolution of $Q$ from minimal free resolutions of $M$ and $T$ or by extracting a minimal free resolution of $M$ from the mapping cone of $\eta_{\bullet}: F_{\bullet} \rightarrow L_{\bullet}$, where $F_{\bullet}, L_{\bullet}$ are minimal free resolutions of $Q$ and $T$, respectively. (For details of such basic constructions, the reader is referred to [3].)

Remark. Since $\operatorname{grade}_{R} T=\operatorname{pd}_{R} T=r, T$ is perfect. It can be easily checked from the above construction that $\operatorname{grade}_{\bar{R}} Q=0$, and since $\operatorname{pd}_{\bar{R}} Q<$ $\infty, \operatorname{support}_{\bar{R}} Q=\operatorname{Spec}(\bar{R})$. Moreover, the strong intersection conjecture (see [12], [15]) is valid for both $Q$ and $T$. For details on this observation we refer the reader to $[15$, Section 4 , Chapitre II].

\section{$\S 2$.}

LEMMA 2.1. Let $(R, m)$ be a local ring of dimension of $n$. Assume that the order ideal conjecture is valid for local rings of dimension $(n-1)$. Then, for the validity of the order ideal conjecture on $R$, it is enough to prove the validity of the assertion for the first syzygies of modules of finite projection. In particular, for cyclic modules of finite projective dimension over $R$, it is enough to prove that every minimal generator of any ideal in $R$ of finite projective dimension over $R$ is a nonzero divisor in $R$.

Proof. Let $\left(F_{\bullet}, d_{\bullet}\right)$ be a minimal free resolution of $M$ where $F_{i}=R^{r_{i}}$ for $i \geq 0$. Let $S_{i}$ denote the $i$ th syzygy of $M$ for $i \geq 1$, and let $\beta$ be a minimal generator of $S_{i}$ for $i>1$. Then $\beta=d_{i}(e)$ for some free generator $e$ of $F_{i}$, and we have $\beta=\left(\begin{array}{c}a_{1} \\ \vdots \\ a_{r_{i-1}}\end{array}\right) \in R^{r_{i-1}}$. Let $J$ denote the ideal generated by $a_{1}, \ldots, a_{r_{i-1}}$. Let $x$ be a nonzero divisor on $R$; then $x$ is a nonzero divisor on 
$S_{1}$. Let $\bar{R}=R / x R$, and let $\bar{S}_{i}=S_{i} / x S_{i}, i \geq 1$. We have that $\bar{S}_{i}$ is of finite projective dimension over $\bar{R}$, and $\left.\bar{S}_{i}=\operatorname{Syz}_{\bar{R}} \frac{-1}{(} \bar{S}_{1}\right)$ for $i>1$. By induction hypothesis, $\operatorname{grade}_{\bar{R}}(J+x R) / x R \geq(i-1)$; this implies that $\operatorname{grade}_{R} J \geq(i-$ $1)$. Let $y \in J$ be a nonzero divisor on $R$. Let $\tilde{R}=R / y R$, and let $\tilde{S}_{i}=S_{i} / y S_{i}$ for $i \geq 1$. Then, again by arguing as above, $\operatorname{grade}_{\tilde{R}} J /(y) \geq(i-1)$. Hence, $\operatorname{grade}_{R} J \geq i$. The second assertion now follows readily.

Lemma 2.2. Let $M$ be a finitely generated module of finite projective dimension over a local ring $(R, m)$. Suppose that $\operatorname{rank}_{R} M=s$. Then there exists a free submodule $F=R^{s}$, generated by a part of a minimal set of generators of $M$, such that $M / F$ has positive grade.

Proof. We induct on $s$. Since $\operatorname{pd}_{R} M$ is finite, if $s=0$, then for every associated prime $P$ of $R, M_{P}=0$, and hence grade $_{R} M>0$. Now suppose that $s>0$. Since $\operatorname{pd}_{R} M$ is finite, by basic element method (see [8, Lemma 2.1]) there exists a minimal generator $\alpha$ of $M$ such that the image of $\alpha$ is a part of a basis of $M_{P}$ for every associate prime $P$ of $R$. Hence, we have a short exact sequence

$$
0 \rightarrow R \rightarrow M \stackrel{\phi}{\longrightarrow} M^{\prime} \rightarrow 0 \quad(1 \rightarrow \alpha)
$$

Then $\operatorname{pd}_{R} M^{\prime}<\infty$ and $\operatorname{rank}_{R} M^{\prime}=\operatorname{rank}_{R} M-1=s-1$. By induction, there exists a free submodule $F^{\prime}=R^{s-1}$ of $M^{\prime}$, generated by a part of a minimal set of generators of $M^{\prime}$, such that $M^{\prime \prime}=M^{\prime} / F^{\prime}$ has positive grade. We have the following short exact sequence:

$$
0 \rightarrow F^{\prime} \stackrel{i}{\longrightarrow} M^{\prime} \stackrel{\psi}{\longrightarrow} M^{\prime \prime} \rightarrow 0
$$

Since $F^{\prime}$ is free, we can lift $i: F^{\prime} \rightarrow M^{\prime}$ to $\eta: F^{\prime} \rightarrow M$ such that $\phi \cdot \eta=i$. Let $\theta=\psi \cdot \phi$. It can be easily checked that $\theta$ is surjective and that $\operatorname{Ker} \theta=R \oplus F^{\prime}$. Hence the lemma follows.

Our next theorem reduces the order ideal conjecture to the assertion that every minimal generator of a certain class of ideals of finite projective dimension must be a nonzero divisor.

TheOREM 2.3. Let $(R, m)$ be a local ring of dimension $n$. Assume that the order ideal conjecture is valid for local rings of dimension $(n-1)$. Then, for the validity of the order ideal conjecture over $R$, it is enough to prove that every minimal generator of any ideal of grade 2 , height 2 , and of finite projective dimension over $R$ is a nonzero divisor in $R$. 
Proof. Let $M$ be a finitely generated module of finite projective dimension over $R$. Due to Lemma 2.1, for the validity of the order ideal conjecture it is enough to consider minimal generators of $S=\operatorname{Syz}_{R}^{1}(M)$. If $\operatorname{grade}_{R} M=0$, then $\operatorname{ann}_{R} M=0$. Let $s_{0}=\operatorname{rank}_{R} M$. By Lemma 2.2, there exists a free submodule $F=R^{s_{0}}$ generated by a part of a minimal set of generators of $M$ such that $M^{\prime}=M / F$ has positive grade. From the commutative diagram

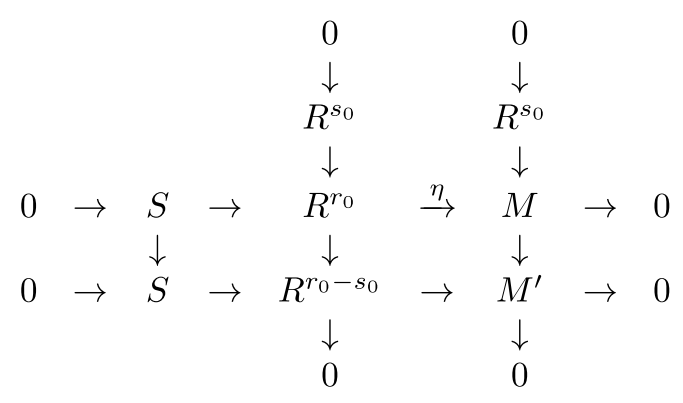

it follows that, without any loss of generality, we can assume that $\operatorname{grade}_{R} M>0$. Let $x$ be a nonzero divisor in $\operatorname{ann}_{R} M$. By Corollary 1.3 we can assume that $M$ has finite projective dimension over $R / x R$ and that $\operatorname{support}(M)=\operatorname{support}(R / x R)$. Let $\bar{R}=R / x R$, and let $\bar{S}=S / x S$. By tensoring $0 \rightarrow S \rightarrow R^{r_{0}} \rightarrow M \rightarrow 0$ with $\bar{R}$, we obtain the short exact sequences

$$
0 \rightarrow M \stackrel{\theta}{\rightarrow} \bar{S} \rightarrow T \rightarrow 0, \quad 0 \rightarrow T \rightarrow \bar{R}^{r_{0}} \rightarrow M \rightarrow 0
$$

where $T=\operatorname{Syz} \frac{1}{R}(M)$. If $\gamma$ is a minimal generator of $M$, and if $e \in R^{r_{0}}$, a free generator of $R^{r_{0}}$, is such that $\eta(e)=\gamma$, then $\theta(\gamma)=\operatorname{Im}(x e)$ in $\bar{S}$. Let $\alpha_{i} \in S$ denote the lifts of a minimal set of generators $\bar{\alpha}_{i}, 1 \leq i \leq h$, of $T$. By induction, grade $\mathcal{O}_{T, \bar{R}}\left(\overline{\alpha_{i}}\right) \geq 1$; hence, grade $\mathcal{O}_{S}\left(\alpha_{i}\right) \geq 1$ for $1 \leq i \leq h$. Due to the exact sequences in (2) we obtain a minimal set of generators $x e_{1}, \ldots, x e_{a}, \alpha_{1}, \ldots, \alpha_{h}$ of $S$ where $e_{1}, \ldots, e_{a}$ form a part of a basis of $R^{r_{0}}$. If $\left(F_{\bullet}, d_{\bullet}\right)\left(F_{i}=R^{r_{i}}\right)$ denote a minimal free resolution of $M$ over $R$, then there exist $\tilde{e}_{1}, \ldots, \tilde{e}_{a}, \ldots, \tilde{e}_{a+j}, \ldots$ a basis of $R^{r_{1}}$ such that $d_{1}\left(\tilde{e}_{i}\right)=x e_{i}, 1 \leq i \leq a$, and $d_{1}\left(\tilde{e}_{a+j}\right)=\alpha_{j}, 1 \leq j \leq h$. Any minimal generator of $S$ is of the form $\sum_{i=1}^{a} c_{i} x e_{i}+\sum_{j=1}^{h} d_{j} \alpha_{j}$, where at least one of $c_{i}$ 's or $d_{j}$ 's is a unit. If any $d_{j}$ in the above expression is a unit, then we are done by induction. Thus, it is easy to check that, in order to show that for any minimal generator $\beta$ of $S$, grade $\mathcal{O}_{S}(\beta) \geq 1$, it is enough to consider $\beta=x e-\sum \lambda_{i} \alpha_{i}$, where $e \in\left\{e_{1}, \ldots, e_{a}\right\}, \sum \lambda_{i} \bar{\alpha}_{i} \neq 0$ in $T$. Due to the second exact sequence in (2), 
if any $\lambda_{i}$ is a unit, then we are done by induction. Hence, we can assume that all $\lambda_{i} \in m$ in the above expression of $\beta$. Let $t=\operatorname{rank}_{\bar{R}} M$. By arguing as in (1) we obtain the following commutative diagram:

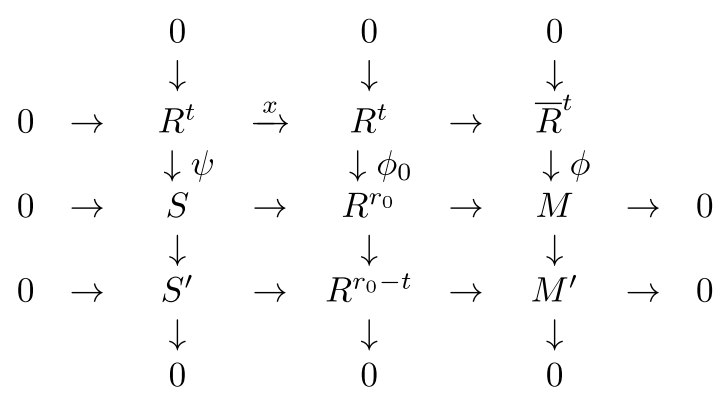

Let $\left\{\bar{e}_{j}\right\}, 1 \leq j \leq t$, denote a basis of $\bar{R}^{t}$ such that $\gamma_{j}=\phi\left(\bar{e}_{j}\right), 1 \leq j \leq t$, in (3) is a part of a minimal set of generators of $M$ (see Lemma 2.2). By commutativity of $(3), \psi\left(e_{j}\right)=x e_{j}, 1 \leq j \leq t$. Note that none of $x e_{j}$ may be a part of $\left\{x e_{1}, \ldots, x e_{a}\right\}$ - part of a minimal basis of $S$ mentioned above. We want to prove the following.

Claim. For any $j, 1 \leq j \leq t$, the grade of the ideal generated by the entries of $\left(x e_{j}-\sum \lambda_{i} \alpha_{i}\right)$ is greater than or equal to 1 (here $x e_{j}=\psi\left(e_{j}\right)$ ).

Proof. Let $\gamma_{1}, \ldots, \gamma_{t}, \gamma_{t+1}, \ldots, \gamma_{r_{0}}$ be a minimal set of generators of $M$ where $\gamma_{j}=\phi\left(\bar{e}_{j}\right), 1 \leq j \leq t$ as above, and $t=\operatorname{rank}_{\bar{R}} M$. Then $\operatorname{grade}_{\bar{R}}\left(M^{\prime}\right) \geq$ 1 ; that is, $\operatorname{grade}_{R}\left(M^{\prime}\right) \geq 2$. Let $y \in \operatorname{ann}_{\bar{R}} M^{\prime}$ be a nonzero divisor in $\bar{R}$; then $\forall k>t$, and $y \gamma_{k}=\sum_{j=1}^{t} a_{k j} \gamma_{j}$; hence, $y e_{k}-\sum a_{k j} e_{j} \in S$ for $k>t$. Let $P \in \operatorname{Ass}_{R}(R)$, and let $q \in \operatorname{Ass}_{\bar{R}}(\bar{R})$ containing $P$. By construction, $q$ is an associated prime of $M$ (recall that ann $\bar{R} M=0$ ); then $M_{q}$ and $T_{q}$ are free $\bar{R}_{q}$ modules of rank $t$ and $r_{0}-t$, respectively. Since $\operatorname{pd}_{R_{q}} M_{q}=1, S_{q}$ is also a free $R_{q}$-module. We have the following short exact sequences:

$$
0 \rightarrow M_{q} \rightarrow \bar{S}_{q} \rightarrow T_{q} \rightarrow 0, \quad 0 \rightarrow T_{q} \rightarrow \bar{R}_{q}^{r_{0}} \rightarrow M_{q} \rightarrow 0
$$

Let $\bar{\beta}_{k}=\bar{e}_{k}-\sum_{j=1}^{t} \frac{\bar{a}_{k j}}{\bar{y}} \bar{e}_{j}$. Then $\left\{\bar{\beta}_{k}\right\}, t+1 \leq k \leq r_{0}$, is a basis of $T_{q}$. Let $\beta_{k}=e_{k}-\sum_{j=1}^{k} \frac{a_{k j}}{y} e_{j}, t+1 \leq k \leq r_{0}$. Then it follows from (4) that $\left\{x e_{1} \ldots\right.$, $\left.x e_{t}, \beta_{t+1}, \ldots, \beta_{r_{0}}\right\}$ form a basis of $S_{q}$. In $S_{q}$, we have

$$
\sum \lambda_{i} \alpha_{i}=\sum_{i=1}^{t} \frac{c_{i}}{b} x e_{i}+\sum_{k=t+1}^{r_{0}} \frac{d_{k}}{b} \beta_{k}, \quad b \notin q .
$$


If $\sum \lambda_{i} \alpha_{i} \notin q S_{q}$, then $\sum \lambda_{i} \bar{\alpha}_{i} \notin q T_{q}$, which implies that some $d_{k} \notin q$. Hence, $\operatorname{Im}\left(x e_{j}-\sum \lambda_{i} \alpha_{i}\right)$ is a minimal generator of $T_{q}$ which is free, and thus $x e_{j}-$ $\sum \lambda_{i} \alpha_{i} \notin P R^{r_{0}}$. Now suppose that $\sum \lambda_{i} \alpha_{i} \in q S_{q}$; then all $c_{i}, d_{i} \in q$ and $x \nmid d_{k}$ for at least one $k$ in (5). Hence, $x e_{j}-\sum \lambda_{i} \alpha_{i}=\left(1-\frac{c_{j}}{b}\right) x e_{j}-\sum_{i \neq j} \frac{c_{i}}{b} x e_{i}-$ $\sum \frac{d_{i k}}{b} \beta_{k}$. Since $1-\frac{q}{b}$ is a unit in $R_{q}, x e_{j}-\sum \lambda_{i} \alpha_{i}$ is a minimal generator of $S_{q}$. Since $S_{q}$ is a free $R_{q}$ module, $x e_{j}-\sum \lambda_{i} \alpha_{i} \notin P R_{q}^{r_{0}}$, and hence $x e_{j}-$ $\sum \lambda_{i} \alpha_{i} \notin P R^{r_{0}}$. This completes the proof of our claim.

Due to the commutative diagram (3) and Lemma 2.2, we can assume, without any loss of generality, that $\operatorname{grade}_{R} M \geq 2$. Now we appeal to the following result due to Smoke [18, Lemma 4.1, Theorem 4.2]. Given a finitely generated $R$-module $M$ of grade greater than or equal to 2 , we can construct an exact sequence

$$
0 \rightarrow M \rightarrow R / I \rightarrow R / J \rightarrow 0
$$

where $R / J$ has a filtration whose successive quotients are isomorphic to cyclic modules of the form $R /(u, v)$, where $\{u, v\}$ form an $R$-sequence. The proof in [18] shows that, by choosing the $R$-sequences of length 2 in $m$ (annihilator of the corresponding module over $R$ ), this exact sequence can be constructed in such a way that, if $\delta: S \rightarrow I$ denotes the corresponding map on first syzygies via (6), then $\delta$ is surjective and $\bar{\delta}: S / m S \rightarrow I / m I$ is an isomorphism. Since $\operatorname{pd}_{R} M<\infty, \operatorname{pd}_{R} R / I<\infty$, and from the construction it follows that height of $I=$ grade of $I=2$. Thus, the proof of our theorem is complete.

Now let us state the following lemma.

Lemma 2.4. Let $(R, m)$ be a local ring, and let $M$ be a finitely generated module of projective dimension $n<\infty$. Let $N$ be an $R$-module such that $\operatorname{ann}_{R} M$ contains an $N$-sequence of length $r$. Then $\operatorname{Tor}_{n-i}^{R}(M, N)=0$ for $0 \leq i<r$.

We leave the proof of this lemma to the reader.

In our next theorem we indicate the vanishing of Tor from an altogether different perspective.

THEOREM 2.5. Let $(R, m)$ be a local ring, and let $M$ be a finitely generated module of finite projective dimension over $R$. Let $\operatorname{pd}_{R} M=h$. Then there exists an $R$-sequence $x_{1}, \ldots, x_{h}$ of length $h$ such that for any $R$-module $N$ for which $x_{1}, \ldots, x_{h}$ form an $N$-sequence, $\operatorname{Tor}_{i}^{R}(M, N)=0$ for $i>0$. 
Proof. If $\operatorname{rank}_{R} M=s_{0}>0$, by Lemma 2.2 there exists a free submodule $F=R^{s_{0}}$ generated by a part of a minimal set of generators of $M$ such that $M^{\prime}=M / F$ has positive grade. From the commutative diagram

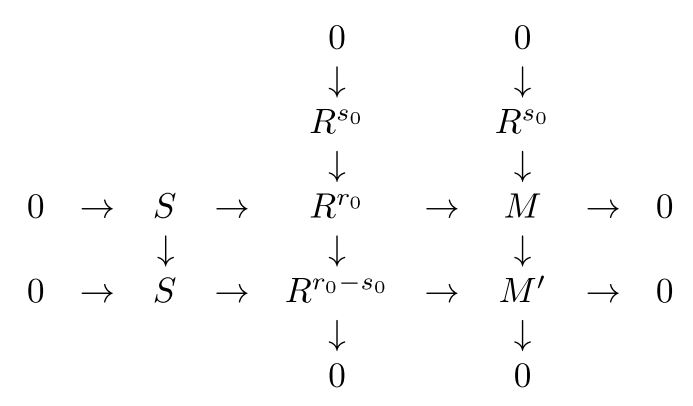

it follows that $S=\operatorname{Syz}_{R}^{1}(M)=\operatorname{Syz}_{R}^{1}\left(M^{\prime}\right)$. If $\operatorname{rank}_{R} M=0$, then $M^{\prime}=M$.

Let $x_{1} \in \operatorname{ann}_{R} M^{\prime}$ be a nonzero divisor on $R$, let $R_{1}=R / x R$, and let $S_{1}=S / x S$. By Theorem 1.2, we have an exact sequence of $R_{1}$-modules

$$
0 \rightarrow M^{\prime} \rightarrow M_{1} \rightarrow V_{1} \rightarrow 0
$$

such that $\operatorname{pd}_{R_{1}} M_{1}<\infty\left(=\operatorname{pd}_{R} M-1\right)$ and $\operatorname{pd}_{R} V_{1}=1$. It is also clear from Corollary 1.3 that $S\left(M_{1}\right)=\operatorname{Syz}_{R}^{1}\left(M_{1}\right)=S \oplus R^{t_{1}}=\operatorname{Syz}_{R}^{1}\left(M^{\prime}\right) \oplus R^{t_{1}}=$ $\operatorname{Syz}_{R}^{1}(M) \oplus R^{t_{1}}$ for some $t_{1} \geq 0$. Tensoring the short exact sequence $0 \rightarrow$ $S\left(M_{1}\right) \rightarrow R^{\ell_{1}} \rightarrow M_{1} \rightarrow 0$ with $R_{1}$, we obtain the following short exact sequences:

$$
0 \rightarrow M_{1} \rightarrow S\left(M_{1}\right) \otimes R_{1} \rightarrow T_{1} \rightarrow 0, \quad 0 \rightarrow T_{1} \rightarrow R_{1}^{\ell_{1}} \rightarrow M_{1} \rightarrow 0 .
$$

Here $T_{1}=\operatorname{Syz}_{R_{1}}^{1}\left(M_{1}\right)$. Recall that $\operatorname{pd}_{R_{1}} M_{1}<\infty$ and that $\operatorname{support}\left(M_{1}\right)=$ Spec $R_{1}$. Now we start with $M_{1}$ over $R_{1}$ and repeat the process described in (1), (2), and (3). We continue this process $(h-2)$ times and obtain an $R$ sequence $x_{1}, \ldots, x_{i}, 1 \leq i \leq h-1$, modules $M_{i}$ of finite projective dimension over $R_{i}=R /\left(x_{1}, \ldots, x_{i}\right)$, and short exact sequences

$$
\begin{aligned}
& 0 \rightarrow M_{i-1}^{\prime} \rightarrow M_{i} \rightarrow V_{i} \rightarrow 0, \\
& 0 \rightarrow F_{i} \rightarrow M_{i} \rightarrow M_{i}^{\prime} \rightarrow 0,
\end{aligned}
$$

where $\operatorname{pd}_{R_{i}} M_{i}=\operatorname{pd}_{R} M-i, \operatorname{support}\left(M_{i}\right)=\operatorname{support}\left(R_{i}\right), \operatorname{pd}_{R_{i-1}} V_{i}=1$, $M_{i-1}^{\prime}=M_{i-1} / F_{i-1}, F_{i-1}$ a free $R_{i-1}$-module as constructed in (1), and $M_{i}^{\prime}$ is a module over $R_{i+1}$. We also have short exact sequences of $R_{i}$-modules

$$
0 \rightarrow M_{i} \rightarrow S\left(M_{i}\right) \otimes R_{i} \rightarrow T_{i} \rightarrow 0, \quad 0 \rightarrow T_{i} \rightarrow R_{i}^{\ell_{i}} \rightarrow M_{i} \rightarrow 0 .
$$


We note that grade $M_{i}=i$ and that $\operatorname{pd}_{R} M_{i}=h$. Let $x_{h} \in m$ be such that $\operatorname{im}\left(x_{h}\right)$ in $R_{h-1}$ is a nonzero divisor contained in $\operatorname{ann}_{R_{h-1}} M_{h-1}^{\prime}$. Then $x_{1}, \ldots, x_{h}$ form an $R$-sequence, and this is our required sequence.

Since projective dimension $\operatorname{pd}_{R} M_{h-1}^{\prime}=h$, if $N$ is an $R$-module such that $x_{1}, \ldots, x_{h}$ form an $N$-sequence, then it follows from Lemma 2.4 that $\operatorname{Tor}_{i}^{R}\left(M_{h-1}^{\prime}, N\right)=0$ for $i>0$. Now it follows from the above short exact sequences starting from $M_{h-1}^{\prime}$ and tracing back to $M$ that $\operatorname{Tor}_{j}^{R}(M, N)=0$ for $j \geq 1$. If $\operatorname{grade}_{R} M=r>0$ and $x_{1}, \ldots, x_{r}$ is an $R$-sequence contained in $\operatorname{ann}_{R} M$, we start with $Q$-an $\bar{R}\left(=R /\left(x_{1}, \ldots, x_{r}\right)\right)$-module as in Theorem 1.2 - and construct an $\bar{R}$-sequence $x_{r+1}, \ldots, x_{h}$ by the above method. Then $x_{1}, \ldots, x_{h}$ form an $R$-sequence satisfying the required vanishing property of Tor. Since $\operatorname{pd}_{R} M<\infty$, if $\operatorname{grade}_{R} M=0$, then $\operatorname{ann}_{R} M=0$, and hence $\operatorname{rank}_{R} M>0$. Thus we are back to diagram (1).

Corollary 2.6. Let $(R, m)$ be a local ring, and let $M$ be a finitely generated module of finite projective dimension $h$ over $R$. Let $x_{1}, \ldots, x_{h}$ be an $R$-sequence as mentioned in the above proposition. Suppose that for every $P \in \operatorname{Ass}_{R}(R)$ there exists an $R / P$-module $N$ such that $x_{1}, \ldots, x_{h}$ form an $N$-sequence and $N \neq m N$. Then, for every minimal generator $\beta$ of $S_{1}=\operatorname{Syz}_{R}^{1}(M), \operatorname{grade} \mathcal{O}_{S_{1}}(\beta) \geq 1$.

Proof. Let $\left(F_{\bullet}, d_{\bullet}\right)$ be a minimal resolution of $M$ over $R$. If possible, let grade $\mathcal{O}_{S_{1}}(\beta)=0$. Then there exists an associated prime $P$ such that $\mathcal{O}_{S_{1}}(\beta) \subset P$. Let $\bar{R}=R / P$, and let $\bar{F} \bullet=F_{\bullet} \otimes R / P$. Consider the sequence

$$
\overline{F_{2}} \stackrel{\overline{d_{2}}}{\longrightarrow} \overline{F_{1}} \stackrel{\overline{d_{1}}}{\longrightarrow} \overline{F_{0}} \rightarrow 0
$$

Since $\mathcal{O}_{S_{1}}(\beta) \subset P, \bar{d}_{1}(\beta)=0$. This implies that, for any $\bar{R}$-module $N$, $\operatorname{Tor}_{1}^{R}(M, N) \neq 0$. However, by hypothesis, there exists an $\bar{R}$-module $N$ such that $x_{1}, \ldots, x_{h}$ form an $N$-sequence. Then, by Theorem 2.5 , we have $\operatorname{Tor}_{j}^{R}(M, N)=0$ for $j>0$, which leads to a contradiction. Hence, $\operatorname{grade} \mathcal{O}_{S_{1}}(\beta) \geq 1$.

Corollary 2.7. Let $(R, m)$ be a local ring, and let $M$ be a finitely generated module of finite projective dimension $h$ over $R$. Let $x_{1}, \ldots, x_{h}$ be an $R$-sequence as mentioned in Theorem 2.5. If $R$ has mixed characteristic $p>0$, we assume that $p, x_{1}, \ldots, x_{h}$ form a part of a system of parameters of $R$. Then for every minimal generator $\beta$ of $S_{1}=\operatorname{Syz}_{R}^{1}(M)$, $\operatorname{grade} \mathcal{O}_{S_{1}}(\beta) \geq 1$. 
Proof. If possible, let grade $\mathcal{O}_{S_{1}}(\beta)=0$. Then there exists an associated prime $P$ of $R$ such that $\mathcal{O}_{S_{1}}(\beta) \subset P$. If $R$ is equicharacteristic, then there exists a big Cohen-Macaulay $R / P$-module $N$ such that $x_{1}, \ldots, x_{h}$ form a regular $N$-sequence. If $R$ has mixed characteristic $p>0$, then there exists a big Cohen-Macaulay $R /(P+p R)$-module $N$ such that $x_{1}, \ldots, x_{h}$ form a regular $N$-sequence. Hence, we are done by Corollary 2.6.

Remark. Due to the existence of Cohen-Macaulay algebras over local domains of dimension less than or equal to 3 (see [14]), it can be checked from the above arguments that finitely generated modules of projective dimension less than or equal to 3 satisfy the order ideal conjecture.

Lemma 2.8. Let $(R, m, K=R / m)$ be an equicharacteristic complete local ring of dimension $d$, and let $x_{1}, \ldots, x_{d}$ be a system of parameters of $R$. Let $M$ be a big Cohen-Macaulay $R$-module such that $\mathbf{x} M \neq M$ and $x_{1}, \ldots, x_{d}$ form a maximal $M$-sequence. Then $\widehat{M}=$ the $m$-adic completion of $M$ is a flat $K\left[\left[x_{1}, \ldots, x_{d}\right]\right]$-module.

Proof. Let $S=K\left[\left[x_{1}, \ldots, x_{d}\right]\right]$. Then $S$ is a complete power series ring in $d$ variables, $R$ is a module-finite extension of $S$, and $\mathbf{x}=\left\{x_{1}, \ldots, x_{d}\right\}$ form a regular system of parameters of $S$. Moreover, $\mathbf{x}$ is $\widehat{M}$-regular (see [3, Theorem 8.5.1]), and $\widehat{M}$ is a balanced big Cohen-Macaulay module (see [3, Corollary 8.5.3]). Hence, $\psi: \frac{\widehat{M}}{\mathbf{x} \widehat{M}}\left[X_{1}, \ldots, X_{d}\right] \rightarrow \bigoplus_{n=0}^{\infty} \frac{\mathbf{x}^{n} \widehat{M}}{\mathbf{x}^{n+1} \widehat{M}}$ is an isomorphism. Since $\mathbf{x}$ is a regular system of parameters of $S, K\left[X_{1}, \ldots, X_{d}\right] \simeq \bigoplus_{n=0}^{\infty} \frac{\mathbf{x}^{n} S}{\mathbf{x}^{n+1} S}$. Hence, $\frac{\mathbf{x}^{n} S}{\mathbf{x}^{n+1} S} \otimes_{K} \widehat{M} / \mathbf{x} \widehat{M} \simeq \frac{\mathbf{x}^{n} \widehat{M}}{\mathbf{x}^{n+1} \widehat{M}}$, and $\frac{\widehat{M}}{\mathbf{x} \widehat{M}}$ is a nonnull vector spacing over $K=S / \mathbf{x} S$. Thus, it follows, by [2, Theorem 1, Section 5.2], that $\widehat{M}$ is $S$-flat.

ThEOREM 2.9 ([11, Theorem 6.9]). Let $(R, m, K)$ be an equicharacteristic complete local ring, and let $N$ be a finitely generated module of finite projective dimension over $R$. Let $I$ be an ideal of height $h$, and let $M$ be a big Cohen-Macaulay module over $R / I$. Let $\widehat{M}$ be the m-adic completion of $M$. Then $\operatorname{Tor}_{i}^{R}(\widehat{M}, N)=0$ for $i>h$.

Proof. For a proof, we refer the reader to [8, Theorem 1.11] or [11], where the existence of a big Cohen-Macaulay $R / I$-module $Q$ such that $\operatorname{Tor}_{i}^{R}(Q, N)=0$, for $i>h$, has been demonstrated. (It was first proved by Foxby [11].) In these proofs, it was required that such a $Q$ be free over a certain complete regular local ring $S$ contained in $R / I$ such that $R / I$ is a 
module-finite extension of $S$. By Lemma 2.8, the completion $\widehat{M}$ of any big Cohen-Macaulay $R / I$ module is flat over certain complete regular subrings of $R / I$. This flatness is enough to ensure the validity of arguments provided in $[8$, Theorem 1.11] or in [11, Theorem 6.9] for proving our assertion.

Next we recall Shimomoto's theorem.

TheOREM 2.10 ([17, Theorem 5.3]). Let $(R, m)$ be a complete local domain of mixed characteristic $p>0$. Then there exists some system of parameters $p, x_{2}, \ldots, x_{d}$ of $R$ and an almost Cohen-Macaulay quasilocal algebra $B$ over $R^{+}$in the sense that

(1) $\left(p, x_{2}, \ldots, x_{d}\right) B \neq B$,

(2) $x_{2}, \ldots, x_{d}$ form a regular sequence on $B / p B$, and

(3) $p$ is not nilpotent in $B$ and the ideal $(0: p)_{\mathbf{B}}$ is annihilated by $p^{\in}$ for any rational $\in>0$.

Actually, Shimomoto's construction shows that such a $B$ can be constructed for any system of parameters of the form $p, x_{2}, \ldots, x_{d}$ of $R$.

Definition. An almost Cohen-Macaulay algebra $B$ as above is called balanced if $B / p B$ is a balanced Cohen-Macaulay algebra.

Now we are ready to prove our final theorem.

THEOREM 2.11. Let $(R, m)$ be a local ring of mixed characteristic $p>0$. We assume that either $p$ is nilpotent or that $p$ is a nonzero divisor in $R$. Let $M$ be a finitely generated module of finite projective dimension over $R$, and let $\beta$ be a minimal generator of $S_{i}$, the ith syzygy of $M$ (minimal), for $i>0$. We have the following.

(a) If $p$ is nilpotent, the order ideal conjecture is valid on $R$.

(b) If $p M=0$, then the grade of $\mathcal{O}_{S_{1}}(\beta) \geq 1$.

(c) Suppose that balanced or complete almost Cohen-Macaulay algebras exist over complete local domains. If $p M=0$ and $p \in m-m^{2}$, then the grade of $\mathcal{O}_{S_{i}}(\beta) \geq i, \forall i \geq 1$.

(d) Assume that every element in $m-m^{2}$ is a nonzero divisor and that the order ideal conjecture is valid over $R / x R$ for any $x \in m-m^{2}$. If $\operatorname{ann}_{R} M \cap\left(m-m^{2}\right) \neq \phi$ or $\operatorname{depth}_{R} M>0$, then $\operatorname{grade} \mathcal{O}_{S_{i}}(\beta) \geq i, \forall i \geq 1$.

Proof. (a) If $p$ is nilpotent, the proof follows immediately by similar arguments as in [9, Theorem 2.4] due to the existence of big Cohen-Macaulay 
modules on equicharacteristic local domains $R / P$ for every prime ideal $P$ in $\operatorname{Spec} R$. One may also use [3, Lemma 9.1.8] to prove the assertion.

(b) Since $p$ is a nonzero divisor on $R$, by Corollary 1.3 we can assume that $\operatorname{pd}_{R / p R} M<\infty$. We write $\bar{R}=R / p R$. Consider the short exact sequence

$$
0 \rightarrow S_{1} \stackrel{g}{\longrightarrow} R^{r_{0}} \stackrel{\eta}{\longrightarrow} M \rightarrow 0
$$

Tensoring this sequence with $\bar{R}$, we obtain the exact sequences

$$
\begin{aligned}
& \mathcal{O} \rightarrow M \stackrel{j}{\longrightarrow} \bar{S}_{1} \stackrel{b}{\longrightarrow} T_{1} \rightarrow 0, \\
& \mathcal{O} \rightarrow T_{1} \stackrel{\gamma}{\longrightarrow} \bar{R}^{r_{0}} \stackrel{\bar{\eta}}{\longrightarrow} M \rightarrow 0,
\end{aligned}
$$

where $T_{1}=\operatorname{Syz} \frac{1}{R}(M)$ and $\bar{S}_{1}=S_{1} \otimes_{R} \bar{R}$. For any minimal generator $\vartheta$ of $M$ we have $\vartheta=\eta(e)$, e being a minimal generator of $R^{r_{0}}$ and $j(\vartheta)=$ class of $p e$ in $\bar{S}$. Recall that if $I$ is an ideal of $R$ such that $\operatorname{grade}_{R} I=0$, then for any nonzero divisor $x$ in $R$, $\operatorname{grade}_{\bar{R}}(I+x R) / x R$ is also 0 . Hence, for any minimal generator $\beta$ of $S_{1}$, if $b(\bar{\beta})$ is a minimal generator of $T_{1}$, then our assertion follows due to the validity of the order ideal conjecture on equicharacteristic local rings. If $b(\bar{\beta})=0$, then $\bar{\beta} \in j(M)$; that is, $\bar{\beta}=j(\vartheta)$, where $\vartheta$ is a minimal generator of $M$. Let $\left\{\bar{\alpha}_{j}\right\}_{1 \leq j \leq t}$ be a minimal set of generators of $T_{1}$, and let $\left\{\alpha_{j}\right\}$ denote a lift of $\left\{\bar{\alpha}_{j}\right\}$ in $S_{1}$. Then there exists a basis $e_{1}, \ldots, e_{i}, \ldots, e_{r_{0}}$ of $R^{r_{0}}$ such that $\left\{p e_{1}, \ldots, p e_{i}, \alpha_{j} s\right\}$ form a minimal set of generators of $S_{1}$. Moreover, to show that, for any minimal generator $\beta$ of $S_{1}$, grade $\mathcal{O}_{S_{1}}(\beta) \geq 1$, it is enough to take $\beta=p e-\sum_{j=1}^{t} \lambda_{j} \alpha_{j}$, where $e \in\left\{e_{1}, \ldots, e_{i}\right\}, \lambda_{j} s \in m$, and $\sum \lambda_{j} \bar{\alpha}_{j} \neq 0$ in $T_{1}$.

If possible, let grade $\mathcal{O}_{S_{1}}(\beta)=0$, that is, let $\mathcal{O}_{S_{1}}(\beta) \subset P$, for some $P \in$ $\operatorname{Ass}_{R}(R)$. Let $\operatorname{pd}_{R} M=h$; then $\operatorname{pd}_{\bar{R}} M=(h-1)$. By Theorem 2.5, corresponding to $M$ over $\bar{R}$, there exists an $\bar{R}$ sequence $\bar{x}_{1}, \ldots, \bar{x}_{h-1}$ satisfying the assertion mentioned in Theorem 2.5. Let $\widetilde{R}=R / P R$, let $\tilde{p}=\operatorname{im}(p)$ in $\widetilde{R}$, and let $\tilde{x}_{i}=\operatorname{im}\left(x_{i}\right)$ in $\widetilde{R}, 1 \leq i \leq h-1$. Then $\tilde{p}, \tilde{x}_{1}, \ldots, \tilde{x}_{h-1}$ form a part of a system of parameters of $\widetilde{R}$. By Corollary 2.7 there exists an almost CohenMacaulay $\widetilde{R}^{+}$-algebra $B$ such that $\left(\tilde{p}, \ldots, \tilde{x}_{h-1}\right) B \neq B$ and $\tilde{x}_{1}, \ldots, \tilde{x}_{h-1}$ form a regular sequence on $B / p B$. Then, by Theorem 2.5 or by [11, Theorem 2.4], we have $\operatorname{Tor}_{j}^{\bar{R}}(M, B / p B)=0$ for $j>0$.

We consider the following part of a minimal resolution $F \bullet$ of $M$ over $R$ :

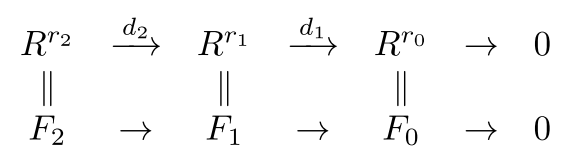


Let $\widetilde{F}_{i}=F_{i} \otimes \widetilde{R}, 0 \leq i \leq 2$. Tensoring the above sequence with $\widetilde{R}$, we obtain a sequence

$$
\widetilde{F}_{2} \stackrel{\tilde{d}_{2}}{\longrightarrow} \widetilde{F}_{1} \stackrel{\tilde{d}_{2}}{\longrightarrow} \widetilde{F}_{0} \rightarrow 0
$$

where $\tilde{\beta}=\operatorname{im}(\beta)$ in $\widetilde{F}_{0}=0$.

Tensoring the above sequence with $B$ and writing $F_{i B}=F_{i} \otimes B$, we have

$$
F_{2 B} \stackrel{d_{2 B}}{\longrightarrow} F_{1 B} \stackrel{d_{1 B}}{\longrightarrow} F_{0 B} \rightarrow 0,
$$

where $\beta_{B}=\operatorname{im} \tilde{\beta}$ in $F_{0 B}=0$. Hence,

$$
p e_{B}=\sum \lambda_{j} \alpha_{j B} \quad \text { in } B^{r_{0}}=F_{0 B}
$$

where $\alpha_{j B}=\operatorname{im}\left(\alpha_{j} \otimes 1_{B}\right)$ in $F_{0 B}, \alpha_{j} \otimes 1_{B} \in S_{1} \otimes B$. Since $\operatorname{Tor}_{j}^{\bar{R}}(M, B / p B)=$ 0 , for $j>0$, tensoring (2) and (3) with $B / p B$ over $\bar{R}$ we obtain the following short exact sequences

$$
0 \rightarrow M \otimes B / p B \stackrel{j \otimes 1_{B / p B}}{\longrightarrow} \overline{S_{1}} \otimes B / p B \rightarrow T_{1} \otimes B / p B \rightarrow 0
$$

and

$$
\mathcal{O} \rightarrow T_{1} \otimes B / p B \rightarrow(B / p B)^{r_{0}} \rightarrow M \otimes B / p B \rightarrow 0
$$

Let $\overline{\alpha_{j B}}=$ the image of $\left(\overline{\alpha_{j}} \otimes 1_{B / p B}\right.$ in $\left.T_{1} \otimes B / p B\right)$ in $(B / p B)^{r_{0}}=\operatorname{im} \alpha_{j B}$ in $(B / p B)^{r_{0}}$. Due to (5) and (7), we have $\sum \lambda_{j} \overline{\alpha_{j B}}=0$ in $(B / p B)^{r_{0}}$, and hence $\sum \lambda_{j}\left(\overline{\alpha_{j}} \otimes 1_{\bar{B}}\right)=0$ in $T_{1} \otimes B / p B$. Since $\lambda_{j} s \in m$, this implies, due to the exact sequence (6) and the definition of $j$ in (2), that

$$
\begin{aligned}
\sum \lambda_{j}\left(\alpha_{j} \otimes 1_{B}\right)= & \sum a_{i}\left(p e_{i} \otimes 1_{B}\right) \\
& +p\left(\sum b_{i}\left(p e_{i} \otimes 1_{B}\right)+\sum \mu_{i}\left(\alpha_{i} \otimes 1_{B}\right)\right)
\end{aligned}
$$

in $S_{1} \otimes B, a_{i} s \in m_{B}$, where $m_{B}$ is the maximal ideal of $B$.

Hence, we have from (5) and (8)

$$
p e_{B}=\sum\left(a_{i}+p b_{i}\right) p e_{i B}+p \sum \mu_{i} \alpha_{i B}
$$

in $B^{r_{0}}$; that is,

$$
p\left\{e_{B}-\left[\sum\left(a_{i}+p b_{i}\right) e_{i B}+\sum \mu_{i} \alpha_{i \beta}\right]\right\}=0 .
$$


Since $a_{i} \in m_{B}$, entries of $\alpha_{i \beta} \in m_{B}, e_{B}-\left[\sum\left(a_{i}+p b_{i}\right) e_{i}+\sum \mu_{i} \alpha_{i B}\right]$ is a free generator of $B^{r_{0}}$; hence, $p$ cannot annihilate it. Thus, $(*)$ leads to a contradiction. Hence, grade $\mathcal{O}_{S_{1}}(\beta)$ must be greater than or equal to 1 .

(c) We assume that $p \in m-m^{2}$ and that $p M=0$. Let $F_{\bullet}: 0 \rightarrow R^{r_{n}} \stackrel{d_{n}}{\longrightarrow}$ $R^{r_{n-1}} \rightarrow \cdots \rightarrow R^{r_{1}} \stackrel{d_{1}}{\rightarrow} R^{r_{0}} \rightarrow 0$ be a minimal free resolution of $M$ over $R$, and let $P_{\bullet}: 0 \rightarrow \bar{R}^{s_{n-1}} \rightarrow \cdots \rightarrow \bar{R}^{s_{1}} \rightarrow \bar{R}^{s_{0}} \rightarrow 0$ be a minimal free resolution of $M$ over $\bar{R}(=R / p R)$. Shamash [16] has shown that $P_{\bullet}$ can be obtained from $F_{\bullet}$ via the homotopy maps $h_{\bullet}: F_{\bullet} \rightarrow F_{\bullet}(+1)$ induced by the 0 -map on $M$ due to multiplication by $p$. Actually, each $F_{i}$, for $i>0$, can be decomposed into two parts: $F_{i}=F_{i}^{\prime} \oplus F_{i}^{\prime \prime}$, where $h_{i}\left(F_{i}^{\prime \prime}\right)=0$, every free generator $e^{\prime}$ of $F_{i-1}^{\prime}$ is such that $e=h_{i-1}\left(e^{\prime}\right)$ is a free generator of $F_{i}^{\prime \prime}$, and $d_{i}(e)=p e^{\prime}-h_{i-2} d_{i-1}\left(e^{\prime}\right)$. Moreover, it follows from Shamash's theorem that (i) there exists $\phi_{\bullet}: P_{\bullet} \rightarrow \overline{F_{\bullet}(+1)}\left(\overline{F_{\bullet}}=F_{\bullet} \otimes_{R} \bar{R}\right)$, where $\phi_{0}$ induces the inclusion map $M=\operatorname{Tor}_{1}^{R}(M, R / p) \stackrel{j}{\hookrightarrow} \bar{S}_{1}$, and (ii) $\phi_{\bullet}$ induces a splitting on each component of $P_{\bullet}$ and $P_{\bullet}(+1)$ can be extracted from the mapping cone of $\phi_{\bullet}$. Let $S_{i}=\operatorname{Syz}_{R}^{i}(M)$, let $T_{i}=\operatorname{Syz} \frac{i}{R}(M)$, and let $\overline{S_{i}}=S_{i} \otimes \bar{R}$. This leads to the following short exact sequences for $i>1$ :

$$
\begin{aligned}
& 0 \rightarrow T_{i-1} \stackrel{\psi}{\longrightarrow} \bar{S}_{i} \stackrel{\eta}{\longrightarrow} T_{i} \rightarrow 0 \\
& 0 \rightarrow T_{i} \rightarrow \bar{R}^{r_{i-1}} \rightarrow T_{i-1} \rightarrow 0
\end{aligned}
$$

where $\psi$ is induced by $\phi_{\bullet}$ and $\psi\left(\operatorname{Im} \bar{e}^{\prime}\right)=\overline{p e-h_{i-2} d_{i-1}\left(e^{\prime}\right)}$ is a minimal generator of $\overline{S_{i}}$ due to the splitting property of $\phi_{\bullet}$. Let $\gamma=p e^{\prime}-h_{i-2} d_{i-1}\left(e^{\prime}\right)$, and let

$$
\bar{\gamma}=\overline{p e^{\prime}-h_{i-2} d_{i-1}\left(e^{\prime}\right)}
$$

Then $\gamma$ and $\bar{\gamma}$ are minimal generators of $S_{i}$ and $\overline{S_{i}}$, respectively.

Claim. In the above situation, grade $\mathcal{O}_{S_{i}}(\gamma) \geq i$.

Proof. If possible let $P$ be a prime ideal of height $(i-1)$ containing $\mathcal{O}_{S_{i}}(\gamma)$. Since $e^{\prime} \in F_{i-1}^{\prime}$ and $h_{i-2}\left(F_{i-2}\right)=F_{i-1}^{\prime \prime}$, it follows that $p \in P$. Let $p, x_{2}, \ldots, x_{i-1}$ be a maximal $R$-sequence contained in $\mathcal{O}_{S_{i}}(\gamma)$-we denote it by $\mathbf{x}$. Then $\operatorname{Tor}_{j}^{R}(M, R / \mathbf{x})=0$ for $j \geq i$. Let $R^{\prime}=R / \mathbf{x}$, let $S_{i}^{\prime}=S_{i} \otimes R^{\prime}$, and so forth. We have an exact sequence

$$
0 \rightarrow S_{i}^{\prime} \rightarrow R^{\prime r_{i-1}} \rightarrow S_{i-1}^{\prime} \rightarrow 0
$$


Then $S_{i-1}^{\prime}$ is a module of finite projective dimension over $R^{\prime}$, and $S_{i}^{\prime}=$ $\operatorname{Syz}_{R^{\prime}}^{\prime}\left(S_{i-1}^{\prime}\right)$ has a minimal generator $\gamma^{\prime}=\operatorname{im} \gamma$ in $S_{i}^{\prime}$ such that $\mathcal{O}_{S_{i}^{\prime}}\left(\gamma^{\prime}\right)$ has grade 0 in $R^{\prime}$. This contradicts part (b) of our theorem, and hence the claim is established.

Let $\left\{\bar{\alpha}_{j}\right\}$ form a minimal set of generators of $T_{i}$, and let $\left\{\alpha_{j}\right\}$ denote their lifts in $S_{i}$. Since characteristic of $\bar{R}=p>0$, by the theorem by Evans and Griffith [9, Theorem 2.4], $\operatorname{grade}_{\bar{R}}\left(\mathcal{O}_{T_{i}}\left(\bar{\alpha}_{j}\right)\right) \geq i$. Since $\left(1^{\prime}\right) \otimes R / m$ is exact due to the above claim, for the purpose of proving our theorem it would be enough to establish that, for every minimal generator $\beta$ of $S_{i}$ of the form $\beta=\gamma-\sum \lambda_{j} \alpha_{j}$, grade $\mathcal{O}_{S_{i}}(\beta) \geq i$, where $\bar{\gamma}=\psi\left(\operatorname{im} \overline{e^{\prime}}\right)$ for some free generator $e^{\prime}$ for $F_{i-1}$ and $\lambda_{j} s \in m$. If possible, let $\mathcal{O}_{S_{i}}(\beta) \subset P$ - a prime ideal of height $(i-1)$. If $p \in P$, there exists a maximal Cohen-Macaulay $R / P$ algebra $\left((R / P)^{+}\right.$-algebra) $\bar{B}$. If $p \notin P$, then by assumption there exists a balanced almost Cohen-Macaulay $R / P$-algebra $\left((R / P)^{+}\right.$-algebra) $B$ such that $p$ is not nilpotent on $B$ and such that $\bar{B}=B / p B$ is a maximal CohenMacaulay algebra over $R /(P+p R)$. Hence, in either case, by [11, Theorem 2.4], $\operatorname{Tor}_{j}^{\bar{R}}(M, \bar{B})=0$ for $j \geq i$. Tensoring $\left(1^{\prime}\right)$ and $\left(2^{\prime}\right)$ with $\bar{B}$, we get the exact sequences

$$
\mathcal{O} \rightarrow T_{i-1} \otimes \bar{B} \stackrel{\psi \otimes 1_{\bar{B}}}{\longrightarrow} \bar{S}_{i} \otimes \bar{B} \rightarrow T_{i} \otimes \bar{B} \rightarrow 0
$$

and

$$
\mathcal{O} \rightarrow T_{i} \otimes \bar{B} \rightarrow \bar{B}^{r_{i-1}} \rightarrow T_{i-1} \otimes \bar{B} \rightarrow 0
$$

Let $e$ be a free generator of $F_{i}$ such that $d_{i}(e)=\beta$.

From $F_{\bullet}$, we consider the exact sequence

$$
F_{i+1} \stackrel{d_{i+1}}{\longrightarrow} F_{i} \stackrel{d_{i}}{\longrightarrow} F_{i-1}
$$

Let $\widetilde{R}=R / P$, and let $\widetilde{F}_{i}=F_{i} \otimes_{R} \widetilde{R}$. Tensoring the above sequence with $\widetilde{R}$, we obtain a complex

$$
\widetilde{F}_{i+1} \stackrel{\tilde{d}_{i+1}}{\longrightarrow} \widetilde{F}_{i} \stackrel{\tilde{d}_{i}}{\longrightarrow} \widetilde{F}_{i-1}
$$

where $\tilde{d}_{i}(\tilde{e})=0$. Let $e_{B}=e \otimes 1_{B}$. Then in the complex $F_{i+1} \otimes B \rightarrow F_{i} \otimes$ $B \rightarrow F_{i-1} \otimes B$, we have $d_{i B}\left(e_{B}\right)=0$. Let $\gamma_{B}=\operatorname{im}\left(\gamma \otimes 1_{B}\right)$, and let $\alpha_{j B}=$ $\operatorname{im}\left(\alpha_{j} \otimes 1_{B}\right)$ in $B^{r_{i-1}}=F_{i-1} \otimes B$. Then

$$
d_{i B}\left(e_{B}\right)=0 \Rightarrow \gamma_{B}-\sum \lambda_{j} \alpha_{j B}=0
$$


Let $\bar{\alpha}_{j B}=\operatorname{Im} \alpha_{j B}$ in $\bar{B}^{r_{i-1}}$. Due to $\left(5^{\prime}\right)$ and $\left(6^{\prime}\right)$, we have $\sum \lambda_{j} \bar{\alpha}_{j B}=0$ in $\bar{B}^{\gamma_{i-1}}$; hence, $\sum \lambda_{j}\left(\bar{\alpha}_{j} \otimes 1_{B}\right)=0$ in $T_{i} \otimes \bar{B}$. Since $\lambda_{j} s \in m$, this implies, due to the exact sequence $\left(6^{\prime}\right)$ and definition of $\psi$ in $\left(1^{\prime}\right)$, that

$$
\begin{aligned}
\sum \lambda_{j}\left(\alpha_{j} \otimes 1_{B}\right)= & \sum a_{i}\left(\gamma_{i} \otimes 1_{B}\right) \\
& +p\left(\sum b_{i}\left(\gamma_{i} \otimes 1_{B}\right)+\sum \mu_{j}\left(\alpha_{j} \otimes 1_{B}\right)\right)
\end{aligned}
$$

in $S_{i} \otimes B, a_{i} s \in m_{B}$. Hence, from $\left(5^{\prime}\right)$ we have

$$
\gamma_{B}=\sum\left(a_{i}+p b_{i}\right)\left(\gamma_{i B}\right)+p \sum \mu_{j} \alpha_{j B}
$$

in $B^{r_{i}-1}$. Since $\gamma_{B}=p e_{B}^{\prime}-h_{i-2} d_{i-1}\left(e_{B}^{\prime}\right)$ and $\gamma_{i B}$ 's also have similar expressions, comparing the $e_{B}^{\prime}$ th coordinate in $B^{r_{i-1}}$, we obtain from above

$$
p\left[e_{B}^{\prime}-\sum\left(a_{i}+p b_{i}\right) e_{B^{\prime}}-\sum \delta_{j}\right]=0
$$

where $\delta_{j}$ is the $e_{B}^{\prime}$ th coordinate of $\mu_{j} \alpha_{j B}$. Since $a_{i}, \delta_{j} \in m_{B}$, the term within brackets in (**) is a free generator of $B^{r_{i-1}}$, and hence $p$ cannot annihilate it. Thus, (**) leads to a contradiction. Hence, grade $\mathcal{O}_{S_{i}}(\beta) \geq i$, and our proof is complete.

(d) We assume that every element in $m-m^{2}$ is a nonzero divisor and that for any such element $x$ the order ideal conjecture is valid for $R / x R$. Let $M$ be a finitely generated module of finite projective dimension such that either $\operatorname{ann}_{R} M \cap\left(m-m^{2}\right) \neq \phi$ or $\operatorname{depth}_{R} M>0$.

First, let us assume that $\operatorname{ann}_{R} M \cap\left(m-m^{2}\right) \neq \phi$. Let $x \in \operatorname{ann}_{R} M \cap(m-$ $m^{2}$ ), and let $\bar{R}=R / x R$. Since $\operatorname{pd}_{R} M<\infty$ and $x \in m-m^{2}, \operatorname{pd}_{\bar{R}} M<\infty$. By hypothesis, $M$ satisfies the order ideal conjecture as an $\bar{R}$-module. Let $\left(F_{\bullet}, d_{\bullet}\right)$ and $\left(P_{\bullet}, S_{\bullet}\right)$ denote minimal free resolutions of $M$ over $R$ and $\bar{R}$, respectively; let $S_{i}=\operatorname{Syz}_{R}^{i}(M)$, and let $T_{i}=\operatorname{Syz}_{\bar{R}}(M)$. Arguing as in the proof of part (b) of the theorem $((1),(2),(3)$, etc.), we construct a minimal set of generators $\left\{x e_{1}, \ldots, x e_{i}, \alpha_{j} s\right\}$ such that $\left\{e_{1}, \ldots, e_{j}\right\}$ form a part of a basis of $F_{0}$, and $\bar{\alpha}_{j}\left(=\operatorname{im} \alpha_{j}\right.$ in $\left.T_{1}\right)$ form a minimal set of generators of $T_{1}$. To show that, for any minimal generators $\beta$ of $S_{1}$, grade $\mathcal{O}_{S_{1}}(\beta) \geq 1$, due to inductive hypothesis, it is enough to take $\beta=x e-\sum \lambda_{i} \alpha_{i}, e \in\left\{e_{1}, \ldots, e_{i}\right\}$, and $\lambda_{i} \in m$. Then $\beta_{e}=$ the eth coordinate of $\beta=x-\sum \lambda_{i} \alpha_{i e}$. Since $\lambda_{i} s \in m$, $\alpha_{i e} s \in m$, and $x \in m-m^{2}$, we have $\beta_{e} \neq 0, \beta_{e} \in m-m^{2}$, and hence by assumption $\beta_{e}$ is a nonzero divisor in $R$. Thus, the conclusion follows for $i=1$. 
Now consider $i>1$. Arguing as in part (c) above we see from $\left(1^{\prime}\right),\left(2^{\prime}\right)$, $\left(3^{\prime}\right)$, and so on, that it is enough to consider a minimal generator $\beta$ of $S_{i}$ of the form $\beta=\gamma-\Sigma \lambda_{i} \alpha_{i}$, where $\gamma=x e^{\prime}-h_{i-2} h_{i-1}\left(e^{\prime}\right)=d_{i}(e), e=h_{i-1}\left(e^{\prime}\right)$, $e^{\prime}$ a minimal generator of $F_{i-1}^{\prime}$, and $\bar{\alpha}_{j}\left(=\operatorname{im} \alpha_{j} \in T_{i}\right)$ form a minimal set of generators of $T_{i}$. Similar arguments as in part (c) show that the grade of $\mathcal{O}_{S_{i}}(\gamma) \geq i$ and, by hypothesis, that grade $\mathcal{O}_{T_{i}}\left(\bar{\alpha}_{j}\right) \geq i$. Let $J$ denote the ideal generated by entries of $\beta$. Recall that $F_{i-1}=F_{i-1}^{\prime} \oplus F_{i-1}^{\prime \prime}$, that $e^{\prime}$ is a minimal generator of $F_{i-1}^{\prime}$, and that $h_{i-2} d_{i-1}\left(e^{\prime}\right) \in F_{i-1}^{\prime \prime}$. Let $y=$ the $e^{\prime}$ th coordinate of $\beta=x-\sum \lambda_{i} \alpha_{i e^{\prime}}$; since $\lambda_{i} s \in m$ and $\alpha_{i e^{\prime}} s \in m, y \in m-m^{2}$, and hence, by assumption, $y$ is a nonzero divisor in $R$. Let $\bar{R}=R / y R$, and let $\bar{S}_{i}=S_{i} / y S_{i}$ for $i \geq 1$; then $\bar{S}_{i}=\operatorname{Syz}_{\bar{R}}^{i-1}\left(\bar{S}_{1}\right)$. By hypothesis $J / y R$ has grade $\geq(i-1)$ in $\bar{R}$. This implies that $\operatorname{grade}_{R} J \geq i$, and our proof is complete.

Now assume that $\operatorname{depth}_{R} M>0$. We can find $x \in m-m^{2}$ such that $x$ is a nonzero divisor on $M$. Let $\bar{R}=R / x R$, and let $\bar{M}=M / x M$. Since $\operatorname{pd}_{\bar{R}} \bar{M}=\operatorname{pd}_{R} M<\infty$, by hypothesis, $\bar{M}$ satisfies the order ideal conjecture over $\bar{R}$. Hence $M$ satisfies the order ideal conjecture over $R$.

COROLlary 2.12. Let $(R, m)$ be a local ring of mixed characteristic $p>0$ such that $p$ is a nonzero divisor in $R$. Let $I$ be an ideal of finite projective dimension over $R$. If $p \in I$ or $p$ is a nonzero divisor on $R / I$, then every minimal generator of $I$ is a nonzero divisor in $R$. In particular, if $P$ is a prime ideal of finite projective dimension over $R$, then every minimal generator of $P$ is a nonzero divisor.

Proof. If $p \in I$, the proof follows from Theorem 2.11(b); if $p$ is a nonzero divisor on $R / I$, the result follows from the validity of the order ideal conjecture on $R / p R$ (see [9]).

Corollary 2.13. Let $(R, m)$ be a regular local ring of dimension $n$, and assume that the order ideal conjecture is valid for regular local rings of dimension $(n-1)$. If $M$ is a finitely generated $R$-module such that either $M$ is annihilated by a regular parameter or $\operatorname{depth}_{R} M>0$, then $M$ satisfies the order ideal conjecture.

The proof follows from Theorem 2.11(d).

REMARK. After going through Theorem 1.2 of this paper, one of the referees pointed out that this theorem could be achieved by combining an embedding theorem for modules of finite $G$-dimension due to Christensen and 
Iyengar ("Gorenstein dimension of modules over homomorphisms") with a couple of results from "complete intersection dimension" by Avramov, Gasharov, and Peeva. This author was not aware of this observation. It is also clear from the comments that the other referee and most of the experts are also unaware of this observation. After taking a glance at the proposed proof via this observation, it seems that this approach would require several other notions and is rather technical and not constructive. In comparison, our proof is much more direct and to the point, and it exploits only the Ext characterization of modules of finite projective dimension.

Acknowledgments. I am thankful to the referees for their suggestions toward improving the exposition in this paper.

\section{REFERENCES}

[1] M. Auslander and R.-O. Buchweitz, "The homological theory of maximal CohenMacaulay approximations" in Colloque en l'honneur de Pierre Samuel (Orsay, 1987), Mém. Soc. Math. Fr. (N.S.) 38, Soc. Math. Fr., Marseilles, 1989, 5-37. MR 1044344.

[2] N. Bourbaki, Elements of Mathematics: Commutative Algebra, Addison-Wesley, Reading, MA, 1972. MR 0360549.

[3] W. Bruns and J. Herzog, Cohen-Macaulay Rings, Cambridge Stud. Adv. Math. 39, Cambridge University Press, Cambridge, 1993. MR 1251956.

[4] S. P. Dutta, On the canonical element conjecture, Trans. Amer. Math. Soc. 299, no. 2 (1987), 803-811. MR 0869233. DOI 10.2307/2000525.

[5] - On negativity of higher Euler characteristics, Amer. J. Math. 126 (2004), 1341-1354. MR 2102398.

[6] - The monomial conjecture and order ideals, J. Algebra 383 (2013), 232-241. MR 3037977. DOI 10.1016/j.jalgebra.2013.03.006.

[7] E. G. Evans and P. Griffith, The syzygy problem, Ann. of Math. (2) 114 (1981), 323-333. MR 0632842. DOI 10.2307/1971296.

[8] _ Syzygies, London Math. Soc. Lecture Note Ser. 106, Cambridge University Press, Cambridge, 1985. MR 0811636. DOI 10.1017/CBO9781107325661.

[9] — "Order ideals" in Commutative Algebra (Berkeley, CA, 1987), Math. Sci. Res. Inst. Publ. 15, Springer, New York, 1989, 213-225. MR 1015519. DOI 10.1007/978-1-4612-3660-3_10.

[10] - A graded syzygy theorem in mixed characteristic, Math. Res. Lett. 8 (2001), 605-611. MR 1879804. DOI 10.4310/MRL.2001.v8.n5.a2.

[11] H.-B. Foxby, On the $\mu^{i}$ in a minimal injective resolution, II, Math. Scand. 41 (1977), 19-44. MR 0476801.

[12] M. Hochster, Topics in the Homological Theory of Modules over Commutative Rings, CBMS Reg. Conf. Ser. Math. 24, Amer. Math. Soc., Providence, 1975. MR 0371879.

[13] - Canonical elements in local cohomology modules and the direct summand conjecture, J. Algebra 84 (1983), 503-553. MR 0723406. DOI 10.1016 /0021-8693(83)90092-3.

[14] - Big Cohen-Macaulay algebras in dimension three via Heitmann's theorem, J. Algebra 254 (2002), 395-408. MR 1933876. DOI 10.1016/S0021-8693(02)00086-8. 
[15] C. Peskine and L. Szpiro, Dimension projective finie et cohomologie locale, Publ. Math. Inst. Hautes Études Sci. 42 (1973), 47-119. MR 0374130.

[16] J. Shamash, The Poincaré series of a local ring, J. Algebra 12 (1969), 453-470. MR 0241411.

[17] K. Shimomoto, Almost Cohen-Macaulay algebras in mixed characteristic via Fontaine rings, Illinois J. Math. 55 (2011), 107-125. MR 3006682.

[18] W. Smoke, Perfect modules over Cohen-Macaulay local rings, J. Algebra 106 (1987), 367-375. MR 0880963. DOI 10.1016/0021-8693(87)90002-0.

Department of Mathematics

University of Illinois

Urbana, Illinois 61801

$U S A$

s-dutta@illinois.edu 